Bull. Austral. Math. Soc.

VOL. 52 (1995) [263-278]

\title{
PARTIAL CLONES CONTAINING ALL PERMUTATIONS
}

\author{
Lucien Haddad and Ivo G. Rosenberg
}

For every nonsingleton finite set $A$, we construct three families of partial clones on $A$ that contain all permutations of $A$ and are of continuum cardinality.

\section{INTRODUCTION}

Let $A$ be a finite set. A clone on $A$ is a composition closed set of operations on $A$ containing all the projections. If in this definiton we replace operations by partial operations, then we obtain a partial clone (this and other concepts will be defined precisely in Section 2). The full description of all clones containing all the permutations on $A$ among their unary operations is given in [5]. In particular, it is shown that there are only finitely many such clones. In this paper, we show that this does not hold for partial clones. Actually, the set of such partial clones is of continuum cardinality even for $|A|=2$, in contrast to the well known fact that there are only countably many clones for $|A|=2[7]$. In fact we do more. First we determine all maximal partial clones containing all permutations, and for three of them, we find a family of $2^{\kappa_{0}}$ subclones containing all the permutations. In two cases, such a family is contained in exactly one maximal partial clone. These results show the substantial difference between the lattice of clones and the lattice of partial clones on a finite set.

\section{PRELIMINARIES}

Let $k \geqslant 2$ be an integer and $\mathbf{k}:=\{0,1, \ldots, k-1\}$. For a positive integer $n$, an $n$-ary partial operation on $\mathbf{k}$ is a map $f: \mathcal{D}_{f} \rightarrow \mathbf{k}$ where $\mathcal{D}_{f}$ is a subset of $\mathbf{k}^{\mathbf{n}}$. Let $\mathcal{P}^{(n)}$ denote the set of all $n$-ary partial operations on $\mathbf{k}$ and let $\mathcal{P}:=\bigcup_{n \geqslant 1} \mathcal{P}^{(n)}$. To describe the composition on $\mathcal{P}$, we use Mal'tsev's formalism (see [6]). First we define on the set $\mathcal{P}$ a binary operation $*$, called superposition, as follows. Let $f \in \mathcal{P}^{(n)}$, $g \in \mathcal{P}^{(m)}$ and $r:=m+n-1$. Then $h:=f * g \in \mathcal{P}^{(r)}$ is defined by setting $\mathcal{D}_{h}:=$ $\left\{\left(x_{1}, \ldots, x_{r}\right) \mid\left(x_{1}, \ldots, x_{m}\right) \in \mathcal{D}_{g}\right.$ and $\left.\left(g\left(x_{1}, \ldots, x_{m}\right), x_{m+1}, \ldots, x_{r}\right) \in \mathcal{D}_{f}\right\}$ and for all $\left(x_{1}, \ldots, x_{r}\right) \in \mathcal{D}_{h}$,

$$
h\left(x_{1}, \ldots, x_{r}\right):=f\left(g\left(x_{1}, \ldots, x_{m}\right), x_{m+1}, \ldots, x_{r}\right) .
$$

Received 24th November, 1995.

.The first author was partially supported by NSERC Grant. The second author was partially supported by a NSERC Grant OGP0005407.

Copyright Clearance Centre, Inc. Serial-fee code: 0004-9729/95 \$A2.00+0.00. 
We also define three unary operations $\zeta, \tau$ and $\Delta$ on $\mathcal{P}$ as follows. Let $n>1$ and $f \in \mathcal{P}^{(n)}$. We define $\zeta(f) \in \mathcal{P}^{(n)}, \tau(f) \in \mathcal{P}^{(n)}$ and $\Delta(f) \in \mathcal{P}^{(n)}$ by setting

$$
\begin{aligned}
\mathcal{D}_{\zeta(f)}: & =\left\{\left(a_{1}, a_{2}, \ldots, a_{n}\right) \mid\left(a_{2}, \ldots, a_{n}, a_{1}\right) \in \mathcal{D}_{f}\right\} \\
\mathcal{D}_{\tau(f)}: & =\left\{\left(a_{1}, a_{2}, \ldots, a_{n}\right) \mid\left(a_{2}, a_{1}, a_{3}, \ldots, a_{n}\right) \in \mathcal{D}_{f}\right\} \\
\mathcal{D}_{\Delta(f)}: & =\left\{\left(a_{1}, \ldots, a_{n-1}\right) \mid\left(a_{1}, a_{1}, a_{2}, \ldots, a_{n-1}\right) \in \mathcal{D}_{f}\right\}
\end{aligned}
$$

and

$$
\begin{gathered}
\zeta(f)\left(x_{1}, \ldots, x_{n}\right):=f\left(x_{2}, \ldots, x_{n}, x_{1}\right), \tau(f)\left(x_{1}, \ldots, x_{n}\right):=f\left(x_{2}, x_{1}, \ldots, x_{n}\right), \\
\Delta(f)\left(x_{1}, \ldots, x_{n-1}\right):=f\left(x_{1}, x_{1}, x_{2}, \ldots, x_{n-1}\right),
\end{gathered}
$$

for all $\left(x_{1}, \ldots, x_{n}\right) \in \mathcal{D}_{\zeta(f)}$, all $\left(x_{1}, \ldots, x_{n}\right) \in \mathcal{D}_{\tau(f)}$ and all $\left(x_{1}, \ldots, x_{n-1}\right) \in \mathcal{D}_{\Delta(f)}$. For $n=1$ we put $\zeta(f)=\tau(f)=\Delta(f)=f$. For every positive integer $n$, and every $1 \leqslant i \leqslant n$, let $e_{i}^{n}$ denote the $n$-ary $i$-th projection defined by $e_{i}^{n}\left(x_{1}, \ldots, x_{n}\right):=x_{i}$ for all $\left(x_{1}, \ldots, x_{n}\right) \in \mathbf{k}^{n}$.

The universal algebra

$$
\widetilde{\mathcal{P}}:=\left\langle\mathcal{P}, *, \zeta, \tau, \Delta, e_{1}^{\mathbf{2}}\right\rangle
$$

is called the partial post-iterative algebra on $\mathbf{k}$. A subuniverse (that is, the carrier of a subalgebra) of $\mathcal{P}$ is called a partial clone on $\mathbf{k}$, (for an equivalent definition see [1]). If a partial clone $C$ is contained in the set $O_{\mathbf{k}}$ of all everywhere defined operations, (that is, $f \in \mathcal{P}^{n}$ with $\mathcal{D}_{f}=\mathbf{k}^{n}$ ), then $C$ is called a clone on $\mathbf{k}$.

Let $h \geqslant 1$ and $\rho$ be an $h$-ary relation on $\mathbf{k}$, (that is, $\rho \subseteq \mathbf{k}^{h}$ ), and let $f$ be an $n$-ary partial operation on $\mathbf{k}$. Let $\mathcal{M}\left(\rho, \mathcal{D}_{f}\right)$ consist of all $h \times n$ matrices $A$ whose columns $A_{* j} \in \rho,(j=1, \ldots n)$ and whose rows $A_{i *} \in \mathcal{D}_{f}(i=1, \ldots, h)$. We say that $f$ preserves $\rho$ if for every $A \in \mathcal{M}\left(\rho, \mathcal{D}_{f}\right)$, the $h$-tuple $f(A):=\left(f\left(A_{1_{*}}\right), \ldots, f\left(A_{h^{*}}\right)\right) \in$ $\rho$. Set $\operatorname{Pol}(\rho):=\{f \in \mathcal{P} \mid f$ preserves $\rho\}$.

EXAMPLE. Consider the unary relation (that is, subset of $\mathbf{k}$ ) $\{0\}$. Then

$$
\operatorname{Pol}\{0\}:=\bigcup_{n \geqslant 1}\left\{f \in \mathcal{P}^{(n)}:(0, \ldots, 0) \in \mathcal{D}_{f} \Rightarrow f(0, \ldots, 0)=0\right\} \text {. }
$$

Note that if $\mathcal{M}\left(\rho, \mathcal{D}_{f}\right)=\emptyset$ (that is, if there is no matrix $A$ whose columns are all in $\rho$, and whose rows are all in $\left.\mathcal{D}_{f}\right)$, then trivially $f \in \operatorname{Pol}(\rho)$. Now it is well known that for every relation $\rho$, the set $\mathrm{Pol}(\rho)$ is a partial clone on $\mathbf{k}$ (for example, see [4, 8]) called the partial clone determined by the relation $\rho$.

A $t$-ary relation $\lambda$ is repetition-free if for all $0 \leqslant i<j \leqslant t-1$, there exists $\left(a_{0}, \ldots, a_{t-1}\right) \in \lambda$ with $a_{i} \neq a_{j}$. Note that if there are $0 \leqslant i<j \leqslant t-1$ such that $a_{i}=a_{j}$ for all $\left(a_{0}, \ldots, a_{t-1}\right) \in \lambda$, then $\operatorname{Pol}(\lambda)=\operatorname{Pol}(\sigma)$, where

$$
\sigma:=\left\{\left(x_{0}, \ldots, x_{j-1}, x_{j+1}, \ldots, x_{t-1}\right) \mid\left(x_{0}, \ldots, x_{t-1}\right) \in \lambda\right\} .
$$


Thus when considering partial clones determined by relations, we can restrict our attention to repetition-free relations. The $i$-th component of $\lambda$ is fictitious if $\left(a_{0} \ldots a_{t-1}\right) \in$ $\lambda$ implies that $\left(a_{0}, \ldots, a_{i-1}, x, a_{i+t}, \ldots, a_{t-1}\right) \in \lambda$ for all $x \in \mathbf{k}$. A $t$-ary relation $\lambda$ is called irredundant if it is repetition-free and has no fictitious components. The following result comes from [9]:

LEMma 1. Let $h, t \geqslant 1, \rho$ be an $h$-ary relation, and let $\lambda$ be a $t$-ary irredundant relation on $\mathbf{k}$. Then $\operatorname{Pol}(\rho) \subseteq \operatorname{Pol}(\lambda)$ if and only if for some positive integer $n$ there exist maps $\psi_{i}: \mathbf{h} \rightarrow \mathrm{t}(i=0, \ldots, n-1)$ such that $\mathbf{t}=\bigcup_{i=0}^{n-1} \operatorname{Im} \psi_{i}$ and

$$
\lambda=\left\{\left(x_{0}, \ldots, x_{t-1}\right) \mid\left(x_{\psi_{i}(0)}, \ldots, x_{\psi_{i}(h-1)}\right) \in \rho, i=0, \ldots, n-1\right\} .
$$

Let $E_{h}$ denote the set of all equivalence relations on $\mathbf{h}=\{0, \ldots, h-1\}$ and let $\omega_{h}:=\{(x, x) \mid x \in \mathbf{h}\}$.

Definition: Let $h \geqslant 2$ and $\varepsilon \in E_{h}$. Put

$$
\Delta_{\varepsilon}=\left\{\left(x_{0}, \ldots, x_{h-1}\right) \in \mathbf{k}^{h}:(i, j) \in \varepsilon \Rightarrow x_{i}=x_{j}\right\}
$$

(that is, $\Delta_{\varepsilon}$ consists of all $h$-tuples over $\mathbf{k}$ constant on every block (that is, equivalence class) of $\varepsilon$ ). An $h$-ary relation $\rho$ is diagonal if there exists $\varepsilon \in E_{h}$ such that $\rho=\Delta_{\varepsilon}$. We often denote $\Delta_{e}$ by $\Delta_{X_{1}}, \ldots, X_{n}$, where $X_{1}, \ldots, X_{n}$ are the nonsingleton blocks of $\varepsilon$.

\section{EXAMPLes.}

(1) Let $\varepsilon=\mathbf{h}$. Then

$$
\Delta_{\varepsilon}=\Delta_{h}=\{(x, \ldots, x) \mid x \in \mathbf{k}\} .
$$

(2) Let $h=4$ and let $\varepsilon$ be the equivalence relation on 4 with the two blocks $\{0,3\}$ and $\{1,2\}$. Then

$$
\Delta_{\{0,3\},\{1,2\}}=\{(x, y, y, x) \mid x, y \in \mathbf{k}\} .
$$

The following result, established in [3], characterises the diagonal relations on $\mathbf{k}$.

Lemma 2. Let $h \geqslant 2$ and let $\lambda$ be an $h$-ary relation on $k$. Then $\operatorname{Pol}(\lambda)=\mathcal{P}$ if and only if $\lambda$ is empty or diagonal.

The partial clones on $\mathbf{k}$, ordered by inclusion, form an algebraic lattice $\mathcal{L}_{P}$ [8] in which every meet is the set-theoretical intersection. For $F \subseteq \mathcal{P}$, the partial clone $\langle F\rangle$ generated by $F$, is the intersection of all partial clones containing the set $F$ (or, equivalently, is the set of term operations of the partial algebra $(\mathcal{P} ; F)$ ). 
Definition: Let $h \geqslant 1, \rho$ be an $h$-ary relation on $\mathrm{k}$ and let $S_{h}$ denote the set of all permutations on $\mathbf{h}:=\{0, \ldots, h-1\}$. Set

$$
\Gamma_{h}:=\left\{\left(a_{0}, \ldots, a_{h-1}\right) \in \mathbf{k}^{h} \mid a_{0}, \ldots, a_{h-1} \text { are pairwise distinct }\right\},
$$

and for $\pi \in S_{h}$ let $\rho^{\pi}:=\left\{\left(a_{\pi(0)}, \ldots, a_{\pi(h-1)}\right) \mid\left(a_{0}, \ldots, a_{h-1}\right) \in \rho\right\}$. The relation $\rho$ is said to be

(1) totally symmetric if $\rho^{(\pi)}=\rho$ for every $\pi \in S_{h}$ that is, if $\left(x_{0}, \ldots, x_{h-1}\right) \in \rho \Leftrightarrow\left(x_{\pi(0)}, \ldots, x_{\pi(h-1)}\right) \in \rho$ for all $\pi \in S_{h}$,

(2) totally reflexive if $\mathbf{k}^{h} \backslash \Gamma_{h} \subseteq \rho$, that is, $\left(x_{0}, \ldots, x_{h-1}\right) \in \rho$ whenever $x_{i}=x_{j}$ for some $0 \leqslant i<j \leqslant h-1$.

3. MAXimal PARTial Clones CONTAINing all PERMUTATIONS

A partial clone $C$ is maximal if it is a coatom of $\mathcal{L}_{P}$, that is, if for every partial clone $C^{\prime}$, the inclusion $C \subset C^{\prime}$ implies that $C^{\prime}=\mathcal{P}$. The goal of this section is to find all maximal partial clones containing the permutations. For this, we need to recall the classification of all maximal partial clones on $k$ given in [4]. We start with some terminology:

Definitions: The $h$-ary relation $\rho$ is said to be

(1) areflexive if $\rho \cap \Delta_{\varepsilon}=\emptyset$ for each $\varepsilon \in E_{h}, \varepsilon \neq \omega_{h}$,

(2) quasi-diagonal if $\rho=\sigma \cup \Delta_{e}$ where $\sigma$ is a non-empty areflexive relation, $\varepsilon \in E_{h} \backslash\left\{\omega_{h}\right\}$, and in addition, $\rho \neq \mathbf{k}^{2}$ if $h=2$.

Let

$$
R_{1}:=\Delta_{\{0,1\},\{2,3\}} \cup \Delta_{\{0,3\},\{1,2\}} \cup \Delta_{\{0,2\},\{1,3\}} \quad R_{2}:=\Delta_{\{0,1\},\{2,3\}} \cup \Delta_{\{0,3\},\{1,2\}} .
$$

Suppose now that the $h$-ary relation $\rho$ is of the form

$$
\rho=\sigma \cup\left(\bigcup_{\epsilon \in F}\left(\Delta_{\varepsilon}\right)\right)
$$

where $\sigma$ is an areflexive $h$-ary relation and $F \subset E_{h}$. Put

$$
G_{\sigma}:=\left\{\pi \in S_{h}: \sigma \cap \sigma^{(\pi)} \neq \emptyset\right\} .
$$

The model of $\rho$ is the $h$-ary relation

$$
\begin{aligned}
M(\rho):=\left\{(\pi(0), \ldots, \pi(h-1)): \pi \in G_{\sigma}\right\} \cup & \left(\bigcup_{\varepsilon \in F}\right. \\
& \left.\left\{\left(a_{0}, \ldots, a_{h-1}\right) \in \mathbf{h}^{h} \mid(i, j) \in \varepsilon \Rightarrow a_{i}=a_{j}\right\}\right)
\end{aligned}
$$


on the set $\mathbf{h}=\{0, \ldots, h-1\}$.

Assume that $h, F$ and $\sigma$ satisfy one of the following five cases:

(i) $h \geqslant 2, F=\emptyset$ and $\sigma \neq \emptyset$, that is, $\rho$ is a non-empty $h$-ary areflexive relation;

(ii) $h \geqslant 2, F=\{\varepsilon\}$ where $\varepsilon \neq \omega_{h}, \sigma \neq \emptyset$ and $\sigma \cup \Delta_{\varepsilon} \neq \mathbf{k}^{2}$, that is, $\rho$ is a non-trivial quasi-diagonal $h$-ary relation;

(iii) $h=4$ and $F=\{\{\{0,1\},\{2,3\}\},\{\{0,3\},\{1,2\}\},\{\{0,2\},\{1,3\}\}\}$, that is, $\rho=\sigma \cup R_{1}$, where $\sigma$ is an areflexive 4-ary relation (eventually empty);

(iv) $h=4$ and $F=\{\{\{0,1\},\{2,3\}\},\{\{0,3\},\{1,2\}\}\}$, that is, $\rho=\sigma \cup R_{2}$, where $\sigma$ is an areflexive 4 -ary relation (eventually empty);

(v) $h \geqslant 3, h \leqslant k, F=\bigcup_{0 \leqslant i<j \leqslant h-1}\{i, j\}$ and $\rho \neq \mathbf{k}^{h}$, that is, $\rho$ is a totally reflexive and totally symmetric non-trivial relation.

We say that $\rho$ is coherent if

(1) $G_{\sigma}=\left\{\pi \in S_{h}: \sigma^{(\pi)}=\sigma\right.$ and $\left.\pi(F)=F\right\}$ for the first four cases above and $G_{\sigma}=\left\{\pi \in S_{h}: \sigma^{(\pi)}=\sigma\right\}=S_{h}$ for the fifth case, and

(2) for every non-empty subrelation $\sigma^{\prime}$ of $\sigma$, there exists a relational homomorphism $\psi: \mathbf{k} \rightarrow \mathbf{h}$ from $\sigma^{\prime}$ to $M(\rho)$ such that $\left(\psi\left(i_{0}\right), \ldots, \psi\left(i_{h-1}\right)\right)=$ $(0, \ldots, h-1)$ for at least one $h$-tuple $\left(i_{0}, \ldots, i_{h-1}\right) \in \sigma^{\prime}$.

Let $p_{n}$ denote the partial $n$-ary operation with empty domain. We have:

TheOREM 3. [4] Let $k \geqslant 2$. Every proper partial clone on $k$ extends to a maximal one. If $C$ is a maximal partial clone on $\mathbf{k}$, then either $C=\mathcal{O} \cup\left\{p_{n}: 0<n<\right.$ $\omega\}$ or $C=\operatorname{Pol}(\rho)$ where $\rho$ is one of the following:

(1) an h-ary areflexive or quasi-diagonal relation which is coherent; $h \geqslant 2$,

(2) an h-ary non-trivial totally reflexive and totally symmetric relation; $h \geqslant 3$,

(3) one of the quaternary relations $R_{1}$ or $R_{2}$,

(4) a quaternary coherent relation $\sigma \cup R_{i}$ where $i=1,2$ and $\sigma \neq \emptyset$ is a quaternary areflexive relation.

Consider the maximal partial clone $D:=\mathcal{O} \cup\left\{p_{n}: 0<n<\omega\right\}$. Clearly the partial subclones of $D$ are of the form $C$ or $C \cup\left\{p_{n} \mid 0<n<\omega\right\}$, where $C$ is a clone (of total operations) containing $S_{k}$. The finitely many clones containing $S_{k}$ are described in [5]. We are left with the maximal partial clones of the form Pol $(\rho)$. Earlier we set

$$
\Gamma_{h}:=\left\{\left(x_{0}, \ldots, x_{h-1}\right) \in \mathbf{k}^{h} \mid x_{i} \neq x_{j} \text { for all } 0 \leqslant i<j \leqslant h-1\right\} .
$$

Let $\rho$ be an $h$-ary relation such that $S_{k} \subseteq \operatorname{Pol}(\rho)$. Note that

$$
\left(a_{0}, \ldots, a_{h-1}\right) \in \rho \Longleftrightarrow\left(\pi\left(a_{0}\right), \ldots, \pi\left(a_{h-1}\right)\right) \in \rho \text { for all } \pi \in S_{k} .
$$


Consequently, if $\rho$ meets $\Gamma_{h}$ then $\Gamma_{h} \subseteq \rho$. We have:

Theorem 4. Let $k \geqslant 4$ and $C$ be a maximal partial clone on $\mathbf{k}$ containing $S_{k}$. Then either $C=\mathcal{O} \cup\left\{p_{n}: 0<n<\omega\right\}$ or $C=\operatorname{Pol}(\rho)$ where $\rho$ is one of the following relations on $\mathbf{k}$ :

$$
\Gamma_{k} ; \Gamma_{k} \cup \Delta_{k} ; \mathbf{k}^{h} \backslash \Gamma_{h} \text {, where } h=3, \ldots, k ; R_{1} ; \Gamma_{4} \cup R_{1} \text { or } R_{2} .
$$

Proof: Consider a maximal partial clone $C$ distinct from $\mathcal{O} \cup\left\{p_{n} \mid 0<n<\omega\right\}$ such that $S_{k} \subseteq C$. By Theorem 3, we have $C=\operatorname{Pol}(\rho)$ where $\rho$ is one of the relations described in the theorem. We have

FACT 1. If $\rho$ is an nonempty $h$-ary areflexive relation, then $h=k$ and $\rho=\Gamma_{k}$.

Proof: From the observation above, we deduce that $\rho$ is totally symmetric and thus is equal to $\Gamma_{h}$. Thus its model is the $h$-ary relation $M\left(\Gamma_{h}\right)=\{(\pi(0), \ldots$, $\left.\pi(h-1)) \mid \pi \in S_{h}\right\}$ on the set $\mathbf{h}$. Now let $2 \leqslant h<k$. Then, as $(0, \ldots, h-1)$, $(0,2, \ldots, h-1, h)$ and $(0,1, \ldots, h-2, h) \in \Gamma_{h}$, we see that there is no relational homomorphism $\psi: \mathbf{k} \rightarrow \mathbf{h}$ from $\Gamma_{h}$ to $M\left(\Gamma_{h}\right)$, that is, $\Gamma_{h}$ is not a coherent relation. Moreover, as $M\left(\Gamma_{k}\right)=\Gamma_{k}$, we trivially have that $\Gamma_{k}$ is coherent.

FACT 2. If $\rho$ is an $h$-ary quasi-diagonal relation, then $h=k$ and $\rho=\Gamma_{k} \cup \Delta_{k}$.

PROOF: Let $\rho=\Gamma_{h} \cup \Delta_{\varepsilon}$ for some equivalence relation $\varepsilon$ on $\mathbf{h}$. Suppose that $\rho$ is coherent. Then as shown above, $h=k$. Thus $\rho=\Gamma_{k} \cup \Delta_{\varepsilon}$, and so $\rho$ must be symmetric under every $\pi \in S_{k}$. By condition (1) of the coherence, $\varepsilon=\mathbf{k}$, that is, $\Delta_{\varepsilon}=\Delta_{k}:=\{(x, \ldots, x) \mid x \in \mathbf{k}\}$.

FACT 3. If $\rho$ is an $h$-ary totally reflexive relation, then $\rho=\mathbf{k}^{h} \backslash \Gamma_{h}$.

Proof: Since $\rho$ is totally reflexive, $\mathbf{k}^{h} \backslash \Gamma_{h} \subseteq \rho$. Suppose now that $\rho \cap \Gamma_{h} \neq \emptyset$. By the above remark, $\Gamma_{h} \subseteq \rho$. This gives that $\rho=\mathbf{k}^{h}$ is a diagonal relation, that is, $\operatorname{Pol}(\rho)=\mathcal{P}$, a contradiction.

FACT 4. If $\rho$ is a quaternary relation of the form $\sigma \cup R_{i}$ with $i=1,2$ and $\sigma \subseteq \Gamma_{4}$, then $\rho \in\left\{R_{1}, R_{2}, \Gamma_{4} \cup R_{1}\right\}$.

Proof: As above, $\sigma \neq \emptyset \Rightarrow \sigma=\Gamma_{4}$. Again by the definition of coherence, one can easily verify that the relation $\Gamma_{4} \cup R_{2}$ is not coherent. The proof of Theorem 4 is complete.

Corollary 5. For $k \geqslant 4$ there are $k+4$ maximal partial clones containing all the permutations on $\mathbf{k}$.

On the other hand, there are 8 maximal partial clones on 2 [2], whereby 4 of them contain the two permutations. Moreover, as $\Gamma_{4}=\emptyset$ for $k=3$, there are 7 maximal 
partial clones containing the set $S_{3}$ of all permutations on 3 :

$$
\begin{aligned}
\mathcal{O} \cup\left\{p_{n} \mid 0<n<\omega\right\}, \operatorname{Pol}\left(\Gamma_{3}\right), \operatorname{Pol}\left(\Gamma_{3} \cup \Delta_{3}\right), \operatorname{Pol}\left(3^{3} \backslash \Gamma_{3}\right) & , \operatorname{Pol}\left(R_{1}\right) \\
& \operatorname{Pol}\left(R_{2}\right), \operatorname{Pol}\left(R_{1} \cup \Gamma_{4}\right) .
\end{aligned}
$$

\section{Independent families of partial clones Containing $S_{k}$}

Denote by $\mathcal{S}$ the partial clone $\left\langle S_{k}\right\rangle$ (generated by all the permutations). In this section we show that the intervals of partial clones $\left[\mathcal{S}, \operatorname{Pol}\left(\Gamma_{k}\right)\right],\left[\mathcal{S}, \operatorname{Pol}\left(R_{i}\right)\right], i=1,2$ are of continuum cardinality. For $k=2$, these are all the intervals $[\mathcal{S}, \operatorname{Pol}(\rho)]$ where $\operatorname{Pol}(\rho)$ is a maximal partial clone (see [2]). We start with the following definition motivated by [7].

Definition: A set $\left\{C_{i} \mid i \in I\right\}$ of partial clones on $\mathbf{k}$ is independent if for all subsets $J$ and $L$ of $I$,

$$
\bigcap_{j \in J} C_{j}=\bigcap_{\ell \in L} C_{\ell} \Rightarrow J=L
$$

Lemma 6. A set $\left\{C_{i} \mid i \in I\right\}$ of partial clones on $\mathbf{k}$ is independent if and only if for every $i \in I$, there exists $f_{i} \in \bigcup_{i \in I} C_{i}$, such that for all $j, \ell \in I$, we have that $f_{j} \in C_{\ell} \Longleftrightarrow j \neq \ell$.

Proof: $(\Rightarrow)$ Let $\left\{C_{i} \mid i \in I\right\}$ be independent and let $i \in I$. The set $D:=$ $\bigcap\left\{C_{j} \mid j \in I \backslash\{i\}\right\}$ is nonempty (because otherwise $\emptyset=D=\bigcap_{i \in I} C_{i}$ ). Choose $f_{i} \in D$. $(\Leftarrow)$ Let $\left\{f_{i} \mid i \in I\right\}$ satisfy the condition $J, L \subseteq I$ and let $\bigcap_{j \in J} C_{j}=\bigcap_{\ell \in L} C_{\ell}$. We show that $I \backslash J \subseteq I \backslash L$. Indeed let $h \in I \backslash J$. Then $f_{h} \in \bigcap_{j \in J} C_{j}=\bigcap_{\ell \in L} C_{\ell}$ and so $h \in I \backslash L$. By symmetry $I \backslash L \subseteq I \backslash J$, that is, $I \backslash J=I \backslash L$ and $J=L$.

Corollary 7. If an interval $J=[D, E]$ of partial clones on $\mathbf{k}$ contains an independent set $\left\{C_{i} \mid i \in I\right\}$, then $|J| \geqslant 2^{|I|}$.

I. We find an independent family of partial subclones of $\operatorname{Pol}\left(\Gamma_{k}\right)$. Let $m \geqslant 2$ and let

$$
\rho_{m}:=\left\{\left(x_{0}, \ldots, x_{m-1}\right) \in \mathbf{k}^{m} \mid x_{0}=\ldots=x_{i-1} \neq x_{i} \neq x_{i+1}=\ldots=x_{m-1}=x_{0},\right.
$$

for some $0 \leqslant i \leqslant m-1\}$.

Thus $\left(x_{0}, x_{1}, \ldots, x_{m-1}\right) \in \rho_{m}$ if and only if exactly $m-1$ entries of $\left(x_{0}, x_{1}, \ldots\right.$, $\left.x_{m-1}\right)$ are pairwise equal. For example $\rho_{2}=\left\{(x, y) \in \mathbf{k}^{2} \mid x \neq y\right\}=\Gamma_{2}$. Note that the relation $\rho_{m}$ is totally symmetric. It is easy to verify that $S_{\mathbf{k}} \subseteq \operatorname{Pol}\left(\rho_{m}\right)$ for every $m \geqslant 2$. We show that $\left\{\operatorname{Pol}\left(\rho_{m}\right) \mid m \geqslant 3\right\}$ is an independent set of partial clones on $k$ 
contained in $\operatorname{Pol}\left(\Gamma_{k}\right)$. For this, define for every $n \geqslant 3$, an $n$-ary partial operation $\varphi_{n}$ on $\mathbf{k}$ by setting

$$
\mathcal{D}_{\varphi_{n}}:=\left\{\left(x_{1}, x_{2}, \ldots, x_{n}\right) \in \mathbf{2}^{n} \mid \sum_{i=1}^{n} x_{i}=1\right\}, \text { and } \varphi_{n}\left(x_{1}, x_{2}, \ldots, x_{n}\right):=0 \text {, }
$$

for every $\left(x_{1}, x_{2}, \ldots, x_{n}\right) \in \mathcal{D}_{\varphi_{n}}$. We have:

LEMMA 8. For all $m, n \geqslant 3$,

$$
\varphi_{n} \in \operatorname{Pol}\left(\rho_{m}\right) \Longleftrightarrow m \neq n
$$

Proof: $(\Rightarrow)$ Let $m=n$. We show that $\varphi_{n} \notin \operatorname{Pol}\left(\rho_{n}\right)$. Indeed the identity matrix $I_{n}$ belongs to $\mathcal{M}\left(\rho_{n}, \mathcal{D}_{\varphi_{n}}\right)$ but $\varphi_{n}\left(I_{n}\right)=(0, \ldots, 0) \notin \rho_{n}$.

$(\Leftrightarrow)$ Let $m \neq n$. As noted earlier in Section 1 , to show that $\varphi_{n} \in \operatorname{Pol}\left(\rho_{m}\right)$ it suffices to prove that $\mathcal{M}\left(\rho_{n}, \mathcal{D}_{\varphi_{m}}\right)$ is empty. Consider an $m \times n$ matrix $A=\left(a_{i j}\right) \in$ $\mathcal{M}\left(\rho_{n}, \mathcal{D}_{\varphi_{n}}\right)$. Clearly $A$ is a zero-one matrix. As $A_{i *}=\left(a_{i 1}, \ldots, a_{i n}\right) \in \mathcal{D}_{\varphi_{n}}$, we have that $\sum_{k=1}^{n} a_{i k}=1$, for all $i=1, \ldots, m$. Therefore every row of the matrix $A$ contains exactly one entry 1 while all the other entries are 0 , and so exactly $m$ entries of $A$ are equal to 1 . Now $m>n$ since otherwise at least one column of $A$ would consist of 0 's and thus would not belong to $\rho_{m}$. From $m>n$ it follows that at least one column of $A$, say $A_{* 1}$, contains more than one entry 1 . As $A_{* 1} \in \rho_{m}$, exactly one entry of $A_{* 1}$, say $a_{11}$, is 0 . This gives $a_{i j}=0$ for all $i, j \geqslant 2$. As $n \geqslant 3$ and since the matrix $A$ has exactly $m$ entries equal to 1 , we see that at least one column of $A$ consists of 0 's and so this column does not belong to $\rho_{m}$. This contradiction shows $\mathcal{M}\left(\rho_{m}, \mathcal{D}_{\varphi_{n}}\right)=\emptyset$, and thus $\varphi_{n} \in \operatorname{Pol}\left(\rho_{m}\right)$.

Theorem 9. Let $k \geqslant 2$. The maximal partial clone $\operatorname{Pol}\left(\Gamma_{k}\right)$ on $\mathbf{k}$ contains the independent family $\left\{\mathrm{Pol}\left(\rho_{m}\right) \mid m \geqslant 3\right\}$. Consequently, there are $2^{\aleph_{0}}$ partial subclones of Pol $\left(\Gamma_{k}\right)$ containing $S_{k}$ and contained in no other maximal partial clone.

Proof: Clearly $S_{k} \subseteq \operatorname{Pol}\left(\rho_{i}\right)$ for all $i \geqslant 2$. Moreover

$$
\Gamma_{2}=\rho_{2}=\left\{(x, y) \in \mathbf{k}^{2} \mid(x, x, x, \ldots, x, y) \in \rho_{m}\right\}
$$

and thus by Lemma $1 \operatorname{Pol}\left(\rho_{m}\right) \subseteq \operatorname{Pol}\left(\rho_{2}\right)$, for all $m \geqslant 3$. On the other hand, $\Gamma_{h}=$ $\left\{\left(x_{0}, \ldots, x_{h-1}\right) \mid\left(x_{i}, x_{j}\right) \in \rho_{2}\right.$ for all $\left.0 \leqslant i \leqslant j<h-1\right\}$, and so $\operatorname{Pol}\left(\rho_{2}\right) \subseteq \operatorname{Pol}\left(\Gamma_{k}\right)$. Now Lemmas 6 and 8 show that $\left\{\operatorname{Pol}\left(\rho_{m}\right) \mid m \geqslant 3\right\}$ is an independent family of subclones of $\mathrm{Pol}\left(\Gamma_{k}\right)$. It remains to show that $\mathrm{Pol}\left(\rho_{m}\right)$ is a subclone of no other maximal clone. In order to see that, define $g_{1} \in \mathcal{P}^{(3)}$ by

$$
\begin{array}{r}
\mathcal{D}_{g_{1}}:=\{(0,0,0),(1,0,0),(0,1,0),(1,1,0)\}, g_{1}(0,0,0)=g_{1}(1,0,0)=g_{1}(0,1,0):=0 \\
\text { and } g_{1}(1,1,0):=1
\end{array}
$$


As the last coordinate of every $\widetilde{x} \in \mathcal{D}_{g_{1}}$ is 0 , the set $\mathcal{M}\left(\rho_{m}, \mathcal{D}_{g_{1}}\right)$ is empty, thus $g_{1} \in \operatorname{Pol}\left(\rho_{m}\right)$ for every $m \geqslant 3$. However the $4 \times 3$ matrix

$$
\left(\begin{array}{lll}
1 & 1 & 0 \\
1 & 0 & 0 \\
0 & 0 & 0 \\
0 & 1 & 0
\end{array}\right)
$$

shows that $g_{1} \notin \operatorname{Pol}\left(R_{1}\right) \cup \operatorname{Pol}\left(R_{2}\right)$. Moreover, this same matrix shows that $g_{1} \notin$ $\operatorname{Pol}\left(\Gamma_{4} \cup R_{1}\right)$ whenever $k \geqslant 4$. Now let $k \geqslant 3$. Define the partial binary operation $g_{2}$ by $\mathcal{D}_{g_{2}}:=\mathbf{k} \times\{0\}$ and $g_{2}(0,0):=1 \quad g_{2}(1,0):=\ldots:=g_{2}(k-1,0):=0$. As above, we can show that $g_{2}$ satisfies $g_{2} \in \operatorname{Pol}\left(\rho_{m}\right)$ for all $m \geqslant 2$ while $g_{2} \notin$ $\operatorname{Pol}\left(\Gamma_{k} \cup\{(x, \ldots, x) \mid x \in \mathbf{k}\}\right)$. Finally, one can use the partial ternary operation defined by $\mathcal{D}_{g_{3}}:=\{(1,1,0),(1,0,0), \ldots,(h-1,0,0)\}$ and $g_{3}(1,1,0):=0, g_{3}(1,0,0):=$ $1, g_{3}(2,0,0):=2, \ldots, g_{3}(h-1,0,0):=h-1$ to show that $\operatorname{Pol}\left(\rho_{m}\right) \nsubseteq \operatorname{Pol}\left(\mathbf{k}^{h} \backslash \Gamma_{h}\right)$, for all $3 \leqslant h \leqslant k$ and all $m \geqslant 3$.

II. We construct an independent family of subclones of $\operatorname{Pol}\left(R_{2}\right)$. Let $h \geqslant 1$ and $\mathcal{E}$ be the set of all equivalence relations on $2 h:=\{0, \ldots, 2 h-1\}$ with two blocks each of size $h$. Note that $\mathcal{E}$ has exactly $\left(\begin{array}{c}2 h \\ h\end{array}\right) / 2$ elements. For every $p>1$, define the $2 p$-ary relation $\sigma_{p}$ on $\mathbf{k}$ by

$$
\sigma_{p}:=\bigcup_{\in \in \mathcal{E}} \Delta_{\varepsilon}
$$

For example, $\sigma_{2}=R_{1}$. Note that $\sigma_{p}$ is a totally symmetric relation. Moreover, it is straightforward to see that $S_{\mathrm{k}} \subseteq \operatorname{Pol}\left(\sigma_{2 p}\right)$ for every $p$. For every number $p$, we define the $(p+1)$-ary partial operation $\alpha_{p}$ by

$$
\begin{gathered}
\mathcal{D}_{\boldsymbol{\alpha}_{p}}:=\left\{\left(x_{0}, \ldots, x_{p}\right) \in \mathbf{2}^{p+1} \mid x_{0}+\ldots+x_{p} \in\{0, p\}\right\}, \text { and } \\
\alpha_{p}(0,1, \ldots, 1)=\alpha_{p}(1,0,1 \ldots, 1)=\ldots=\alpha_{p}(1,1, \ldots, 1,0)=1, \alpha_{p}(0,0, \ldots, 0)=0 .
\end{gathered}
$$

Thus $\left|\mathcal{D}_{\alpha_{p+1}}\right|=\boldsymbol{p}+2$.

Lemma 10. Let $F \subseteq \mathbb{N}$ satisfy $p$ divides $q \Longleftrightarrow p=q$, for all $p, q \in F$. Then for all $p, q \in F$

$$
\alpha_{p} \in \operatorname{Pol}\left(\sigma_{q}\right) \Longleftrightarrow p \neq q .
$$

Proof: $(\Rightarrow)$ By contraposition. Let $p=q$ and consider the $2 p \times(p+1)$ matrix $A$ whose first $p+1$ rows are $(1,1, \ldots, 1,0),(1,1, \ldots, 0,1), \ldots,(0,1, \ldots, 1,1)$, and 
whose last $p-1$ rows are $(0,0, \ldots, 0,0)$, that is,

$$
A:=\left(\begin{array}{ccccc}
1 & 1 & \ldots & 1 & 0 \\
1 & 1 & \ldots & 0 & 1 \\
\vdots & \vdots & \ldots & \vdots & \vdots \\
1 & 0 & \ldots & 1 & 1 \\
0 & 1 & \ldots & 1 & 1 \\
0 & 0 & \ldots & 0 & 0 \\
\vdots & \vdots & \ldots & \vdots & \vdots \\
0 & 0 & \ldots & 0 & 0
\end{array}\right)
$$

It is clear that $A \in \mathcal{M}\left(\sigma_{p}, \mathcal{D}_{\alpha_{p}}\right)$ while $\alpha_{p}(A)=(1, \ldots, 1,0, \ldots, 0)$ consisting of $(p+1)$ ones and $(p-1)$ zeros does not belong to $\sigma_{p}$, proving that $\alpha_{p} \notin \operatorname{Pol}\left(\sigma_{p}\right)$.

$(\Leftrightarrow)$ Let $p \neq q \in F$. To show that $\alpha_{p} \in \operatorname{Pol}\left(\sigma_{q}\right)$ consider a matrix $A \in$ $\mathcal{M}\left(\sigma_{q}, \mathcal{D}_{\alpha_{q}}\right)$. Clearly $A$ is a zero-one matrix. We have:

Claim. At least one column of $A$ is constant.

Proof: (of the claim) Suppose to the contrary that no column of $A$ is constant. Then every column sum is $q$ and so $A$ has exactly $q(p+1)$ ones. By the definition of $\mathcal{D}_{\alpha_{p}}$, each row sum of $A$ is either 0 or $p$. Thus $p$ divides $q(p+1)$ and consequently $p$ divides $q$. As $p \neq q$, the number $p$ is a proper divisor of $q$, a contradiction.

Thus $A$ contains at least one constant column. Note that if some column of $A$ is $(1,1, \ldots, 1)$, then no row of $A$ is the zero row and so

$$
\alpha_{p}(A)=(1,1, \ldots, 1) \in \sigma_{q}
$$

Thus we may assume that no column of $A$ is $(1, \ldots, 1)$. Then some column of $A$, say the first, is the zero vector. Clearly all the nonzero rows of $A$ are $(0,1, \ldots, 1)$. If $A$ is the zero matrix, then clearly $\alpha_{p}(A)=(0, \ldots, 0) \in \sigma_{p}$. Thus let $A$ be nonzero. Then $A$ has exactly $q$ rows $(0,1, \ldots, 1)$ and $q$ zero rows; hence $\alpha_{p}(A)$ consists of $q$ ones and $q$ zeros and therefore $\alpha_{p}(A) \in \sigma_{q}$.

Now we can prove:

Theorem 11. Let $k \geqslant 2$. The maximal partial clone $\mathrm{Pol}\left(R_{2}\right)$ on $\mathrm{k}$ contains the independent family $\left\{\operatorname{Pol}\left(\sigma_{p}\right) \mid p\right.$ is an odd prime $\}$. Consequently, there are $2^{\aleph_{0}}$ partial subclones of $\mathrm{Pol}\left(R_{2}\right)$ containing the set $S_{k}$.

ProOF: Let $p \geqslant 3$ and

$$
\lambda:=\left\{\left(x_{1}, x_{2}, x_{3}, x_{4}\right) \in \mathbf{k}^{4} \mid\left(x_{1}, \ldots, x_{1}, x_{2}, x_{3}, \ldots, x_{3}, x_{4}\right) \in \sigma_{p}\right\},
$$


with $p-1$ symbols $x_{1}$ and $p-1$ symbols $x_{3}$. Then by Lemma 1 we have $\operatorname{Pol}\left(\sigma_{p}\right) \subseteq$ $\operatorname{Pol}(\lambda)$. We show that $\lambda=R_{2}$. Let $\left(x_{1}, x_{2}, x_{3}, x_{4}\right) \in R_{2}$. Then either $(i) x_{1}=x_{2}$ and $x_{3}=x_{4}$ or $(i i) x_{1}=x_{4}$ and $x_{2}=x_{3}$. In both cases $\left(x_{1}, \ldots, x_{1}, x_{2}, x_{3}, \ldots, x_{3}, x_{4}\right) \in \sigma_{p}$ and so $R_{2} \subseteq \lambda$. Conversely let $\widetilde{x}=\left(x_{1}, x_{2}, x_{3}, x_{4}\right) \in \lambda$. Then $\tilde{y}=\left(x_{1}, \ldots, x_{1}, x_{2}, x_{3}\right.$, $\left.\ldots, x_{3}, x_{4}\right) \in \sigma_{p}$. Suppose $x_{1}=x_{3}$. Then $\tilde{y}$ has $2 p-2$ equal coordinates. As $2 p-2>p$, clearly $x_{1}=x_{2}=x_{3}=x_{4}$ and $\tilde{x} \in R_{2}$. Then either $x_{1}=x_{2}$ and $x_{3}=x_{4}$ or $x_{1}=x_{4}$ and $x_{2}=x_{3}$, that is, $\left(x_{1}, x_{2}, x_{3}, x_{4}\right) \in R_{2}$, proving the claim. By Lemma 10 , the set $\left\{\mathrm{Pol}\left(\sigma_{p}\right) \mid p\right.$ is odd prime $\}$ is independent.

As for the family $\left\{\operatorname{Pol}\left(\rho_{m}\right) \mid m \geqslant 3\right\}$, we can prove:

Proposition 12. $\operatorname{Pol}\left(R_{2}\right)$ is the unique maximal partial clone on $\mathbf{k}$ that contains the family of partial clones $\left\{\mathrm{Pol}\left(\sigma_{2 p}\right) \mid p \geqslant 3\right\}$.

Proof: The partial ternary operation $\alpha_{2}$ shows that, for every $p>2, \operatorname{Pol}\left(\sigma_{p}\right) \nsubseteq$ $\operatorname{Pol}\left(R_{1}\right) \cup \operatorname{Pol}\left(\Gamma_{4} \cup R_{1}\right)$. Indeed by Lemma $10 \alpha_{2} \in \operatorname{Pol}\left(\sigma_{p}\right)$. Let

$$
A:=\left(\begin{array}{lll}
1 & 1 & 0 \\
1 & 0 & 1 \\
0 & 1 & 1 \\
0 & 0 & 0
\end{array}\right) .
$$

Clearly $A \in \mathcal{M}\left(R_{1}, \mathcal{D}_{\alpha_{2}}\right) \cap \mathcal{M}\left(\Gamma_{4} \cup R_{1}, \mathcal{D}_{\alpha_{2}}\right)$ but $\alpha_{2}(A)=(1,1,1,0)$ is neither in $R_{1}$ nor in $\Gamma_{4} \cup R_{1}$. Moreover the partial clone $\operatorname{Pol}\left(\sigma_{2}\right)$ contains all the constant functions while $\operatorname{Pol}\left(\Gamma_{k}\right)$ does not. Let $k \geqslant 3$. The unary operation $f$ defined by $f(0)=\ldots=f(k-2)=0$ and $f(k-1)=1$ satisfies $f \in \operatorname{Pol}\left(\sigma_{p}\right) \backslash \operatorname{Pol}\left(\Gamma_{k} \cup \Delta_{k}\right)$, because every unary operation preserves $\sigma_{p}$ but only the permutations and the constant function preserve $\Gamma_{k} \cup \Delta_{k}$. Finally, for $3 \leqslant h \leqslant k$, the partial binary operation defined by setting $\mathcal{D}_{g}:=\{(0,1)\} \cup\{(0,0),(1,1), \ldots,(h-2, h-2)\}$ and $g(0,0)=0, g(0,1)=$ $1, g(1,1)=2, \ldots, g(h-2, h-2)=h-1$ satisfies $g \in \operatorname{Pol}\left(\sigma_{p}\right) \backslash \operatorname{Pol}\left(\mathbf{k}^{h} \backslash \Gamma_{h}\right)$. Indeed, let $A \in \mathcal{M}\left(\sigma_{p}, \mathcal{D}_{g}\right)$ contain $n_{0}$ rows $(0,0), n_{1}$ rows $(0,1)$ and $n_{i}$ rows $(i-1, i-1), i=$ $2, \ldots, h-1$. If $n_{0}=0$, then $\left(a_{1}, \ldots, a_{2 p}\right):=g(A)$ belongs to $\sigma_{p}$ because there is a 1-1 correspondance between the first column of $A$ and $\left(a_{1}, \ldots, a_{2 p}\right)$. If $n_{1}=$ 0 , then by a similar argument (using the second column) again $g(A) \in \sigma_{p}$. Thus assume that both $n_{0}$ and $n_{1}$ are positive. From the second column of $A$ we see that $n_{0}=n_{1}=p$, hence $n_{2}=\ldots=n_{h-1}=0$ and $g(A) \in \sigma_{p}$. The matrix $B$ with rows $\{(0,0),(0,1),(1,1), \ldots,(h-2, h-2)\}$, clearly belongs to $\mathcal{M}\left(\mathbf{k}^{h} \backslash \Gamma_{h}, \mathcal{D}_{g}\right)$ but $g(B)=(0,1, \ldots, h-1) \notin \mathbf{k}^{h} \backslash \Gamma_{h}$.

III. We construct an independent family of subclones of $\operatorname{Pol}\left(R_{1}\right) \cap \operatorname{Pol}\left(R_{2}\right)$. For $n \geqslant 3$ let $\zeta_{n}$ consist of all $\left(a_{1}, \ldots, a_{n}\right) \in \mathbf{k}^{n}$ with $a_{1}=\cdots=a_{i-1}=a_{i+1}=\cdots=$ $a_{j-1}=a_{j+1}=\cdots=a_{n}$ and $a_{i}=a_{j}$ for some $1 \leqslant i<j \leqslant n$. Thus $\zeta_{n}$ is the set of 
all constant $n$-tuples over $\mathbf{k}$ and of all 2 -valued $n$-tuples over $\mathbf{k}$ with frequencies 2 and $n-2$. We first show that $\left\{\operatorname{Pol}\left(\zeta_{7}\right), \operatorname{Pol}\left(\zeta_{8}\right), \ldots\right\}$ is an independent family of partial clones on $\mathbf{k}$.

The set $\left.P_{n}:=\{\{p, q\} \mid 1 \leqslant p<q \leqslant n\}\right\}$ can be ordered lexicographically: Set $\{p, q\} \prec\left\{p^{\prime}, q^{\prime}\right\}$ if (i) $p \leqslant p^{\prime}$ and (ii) $q<q^{\prime}$ whenever $p=p^{\prime}$. Denote by $M^{n}$ the following $n \times\left(\begin{array}{c}n \\ 2\end{array}\right)$ zero-one matrix: The columns of $M^{n}=\left(m_{i\{p, q\}}\right)$ are indexed by $\{p, q\} \in P_{n}$ listed in the lexicographic order and $m_{i\{p, q\}}=1$ if $i \in\{p, q\}$ and $m_{i\{p, q\}}=0$ if $i \notin\{p, q\}$. For example,

$$
M^{5}=\left(\begin{array}{llllllllll}
1 & 1 & 1 & 1 & 0 & 0 & 0 & 0 & 0 & 0 \\
1 & 0 & 0 & 0 & 1 & 1 & 1 & 0 & 0 & 0 \\
0 & 1 & 0 & 0 & 1 & 0 & 0 & 1 & 1 & 0 \\
0 & 0 & 1 & 0 & 0 & 1 & 0 & 1 & 0 & 1 \\
0 & 0 & 0 & 1 & 0 & 0 & 1 & 0 & 1 & 1
\end{array}\right) .
$$

For $1 \leqslant i \leqslant n$ and $\{p, q\} \in P_{n}$, denote by $M_{i *}^{n}$ and $M_{*\{p, q\}}^{n}$ the $i$-th row and $\{p, q\}$-th coloumn of $M^{n}$. Define a partial $\left(\begin{array}{l}n \\ 2\end{array}\right)$-ary operation $f_{n}$ on $\mathbf{k}$ as follows. The domain $\mathcal{D}_{n}$ of $f_{n}$ is the set $\left\{M_{1 *}^{n}, \ldots, M_{n *}^{n}\right\}$ of rows of $M^{n}$ and $f\left(M_{1 *}^{n}\right)=1, f\left(M_{2 *}^{n}\right)=\ldots=$ $f\left(M_{n *}^{n}\right)=0$. We need:

LEMMA 13. Let $m, n \geqslant 5$. Then

$$
f_{n} \in \operatorname{Pol}\left(\zeta_{m}\right) \Longleftrightarrow m \neq n
$$

ProOF: For $i=1, \ldots, n$ set $a_{i}:=f_{n}\left(M_{i *}^{n}\right)$.

$(\Rightarrow)$ By contraposition. Let $m=n$. Clearly $M^{n} \in \mathcal{M}\left(\zeta_{n}, \mathcal{D}_{f_{n}}\right)$ but $f_{n}\left(M^{n}\right)=$ $(1,0, \ldots, 0) \notin \zeta_{\boldsymbol{n}}$.

$(\Leftarrow)$ Let $m \neq n$ and let $X$ be an $m \times n$ matrix over $\mathbf{k}$ whose rows belong to $\mathcal{D}_{n}$ and such that $f_{n}(X) \notin \zeta_{m}$. Clearly there exist $1 \leqslant i_{1}, \ldots, i_{m} \leqslant n$ such that $X_{j *}=M_{i^{*}}^{n}$ for all $j=1, \ldots, m$. Set $A:=\left\{i_{j} \mid j=1, \ldots, m\right\}$. (1) First suppose that $A \subset\{1, \ldots, n\}$. Choose $a \in A$ and $b \in\{1, \ldots, n\} \backslash A$. Then the $\{a, b\}$-th column of $X$ contains exactly one 1 - namely $m_{a,\{a, b\}}$ - and $X_{*\{a, b\}} \notin \zeta_{m}$ and we are done.

(2) Thus let $A=\{1, \ldots, n\}$. Then $m \geqslant n$ and so $m>n$ due to the assumption $m \neq n$. It follows that $i_{p}=i_{q}$ for some $1 \leqslant p<q \leqslant m$. In view of $|A|=n \geqslant 5$, clearly $i_{p} \neq i_{r}$ for some $1 \leqslant r \leqslant m$. Set $a:=i_{p}, b:=i_{r}$ and $z:=\{a, b\}$. Then the $z$-th column $c:=X_{* z}$ of $X$ contains at least three 1 's, namely $m_{a z}=1$ twice (in the $p$-th and $q$-th row of $X$ ), and $m_{b z}=1$ once (in the $r$-th row of $X$ ). Suppose now that $c \in \zeta_{m}$. Then by the definition of $\zeta_{m}$, the vector $c$ has at least $m-2$ ones. Now the definition of $M^{n}$ shows that $|A \backslash\{a, b\}| \leqslant 2$ which leads to the contradiction $|A| \leqslant 4$. Thus $c \notin \zeta_{m}$ and so $f_{n} \in \operatorname{Pol}\left(\zeta_{m}\right)$.

Now we have: 
Lemma 14. If $n \geqslant 7$ then $\operatorname{Pol}\left(\zeta_{n}\right) \subseteq \operatorname{Pol}\left(R_{1}\right) \cap \operatorname{Pol}\left(R_{2}\right)$.

PROOF:

(1) First we prove that $\operatorname{Pol}\left(\zeta_{n}\right) \subseteq \operatorname{Pol}\left(R_{1}\right)$. Set

$$
\alpha:=\left\{(x, y, z, t) \in \mathbf{k}^{4} \mid(x, y, z, t, \ldots, t) \in \zeta_{n}\right\} .
$$

Clearly $\operatorname{Pol}\left(\zeta_{n}\right) \subseteq \operatorname{Pol}(\alpha)$ and so it suffices to show

Claim 1. $\alpha=R_{1}$.

Proof: (of the claim). It is quite easy to verify that $R_{1} \subseteq \alpha$; for example, $(a, a, b, b) \in \alpha$ as $(a, a, b, \ldots, b) \in \zeta_{n}$ and so on. Suppose now that $\alpha \nsubseteq R_{1}$ and let $\left(a_{1}, \ldots, a_{4}\right) \in \alpha \backslash R_{1}$. From (1) it follows that $a_{1}, \ldots, a_{4}$ are not pairwise distinct and therefore exactly three of $a_{1}, \ldots, a_{4}$ are equal. However, if $a, b$ are distinct elements of $\mathbf{k}$, then as $n \geqslant 7$, we deduce from (1) that $(a, a, a, b) \notin \alpha$. Similarly, $(a, a, b, a),(a, b, a, a),(b, a, a, a) \notin \alpha$. This contradiction shows that $R_{1} \subseteq \alpha$ and proves our claim.

(2) Now we show that $\operatorname{Pol}\left(\zeta_{n}\right) \subseteq \operatorname{Pol}\left(R_{2}\right)$. Set

$$
\beta:=\left\{(x, y, z, t) \in \mathbf{k}^{4} \mid(x, y, z, t, \ldots, t),(x, x, z, z, t, \ldots, t) \in \zeta_{n}\right\}
$$

Again it is clear that $\operatorname{Pol}\left(\zeta_{\pi}\right) \subseteq \operatorname{Pol}(\beta)$ and so it remains to show:

Claim 2. $\beta=R_{2}$.

Proof: (of the claim) First we show that $R_{2} \subseteq \beta$. Indeed, for $a, b \in \mathbf{k}$ clearly $(a, a, b, b) \in \beta$ due to $(a, a, b, \ldots, b) \in \zeta_{n}$ and similarly $(a, b, b, a) \in \beta$ as $(a, b, b, a, \ldots, a) \in \zeta_{n}$. Conversely, we show that $R_{2} \subseteq \beta$. Observe that, as $(x, y, z, t) \in \beta$ implies $(x, y, z, t, \ldots, t) \in \zeta_{n}$, proceeding as in the proof of Claim 1 , we deduce that $\beta \subseteq R_{1}$. Suppose now that $\beta \nsubseteq R_{2}$, and let $\left(a_{1}, \ldots, a_{4}\right) \in \beta \backslash R_{2}$. Then $a_{1}=a_{3}=a \neq b=a_{2}=a_{4}$ and from (2) we obtain that $(a, a, a, a, b, \ldots, b) \in \zeta_{n}$. As $n \geqslant 7$, this contradicts the definition of $\zeta_{n}$. Thus $\beta=R_{2}$, and the proof of our lemma is complete.

We have shown:

THEOREM 15. The partial clone $C:=\operatorname{Pol}\left(R_{1}\right) \cap \operatorname{Pol}\left(R_{2}\right)$ contains the independent family of clones $\left\{\operatorname{Pol}\left(\zeta_{7}\right), \operatorname{Pol}\left(\zeta_{8}\right), \ldots\right\}$. Consequently there are $2^{\aleph_{0}}$ partial subclones of $C$ containing all the permutations of $\mathbf{k}$.

Furthermore we have:

Proposition 16. Let $k, n \geqslant 3$. Then $\operatorname{Pol}\left(R_{1}\right)$ and $\operatorname{Pol}\left(R_{2}\right)$ are the only maximal partial clones on $\mathbf{k}$ containing $\operatorname{Pol}\left(\zeta_{n}\right)$.

Proof: Each constant unary operation belongs to Pol $\left(\zeta_{n}\right)$ but does not belong to $\mathrm{Pol}\left(\Gamma_{k}\right)$. Let the unary operation $g$ on $\mathbf{k}$ be defined by $g(0):=1$ and $g(x):=0$ 
otherwise. It is easy to see that $g \in \operatorname{Pol}\left(\zeta_{n}\right)$ but clearly $g \notin \operatorname{Pol}\left(\Gamma_{k} \cup \Delta_{k}\right)$. Also, for $k>3$, clearly $(0,1,2,3) \in R_{1} \cap R_{2} \cap\left(\Gamma_{k} \cup R_{1}\right)$ while $(g(0), g(1), g(2), g(3))=$ $(1,0,0,0) \notin R_{1} \cup R_{2} \cup\left(\Gamma_{4} \cup R_{1}\right)$, proving $g$ is in neither $\operatorname{Pol}\left(R_{1}\right), \operatorname{Pol}\left(R_{2}\right)$ nor in $\operatorname{Pol}\left(\Gamma_{4} \cup R_{1}\right)$. We show that $\operatorname{Pol}\left(\zeta_{n}\right) \nsubseteq \operatorname{Pol}\left(k^{h} \backslash \Gamma_{h}\right)$, for $h=3, \ldots, k$. Consider the partial ternary operation $\gamma$ defined by

$$
\mathcal{D}_{\gamma}:=\{(0,0,0),(0,1,1),(1,0,2),(2,2,0)\} \cup\{(i, i, i) \mid i=3, \ldots, h-2\},
$$

and $\gamma(0,0,0):=0, \gamma(0,1,1):=1, \gamma(1,0,2):=2, \gamma(2,2,0):=3, \gamma(i, i, i):=i+1$, $(i=3, \ldots, h-2)$. Clearly

$$
A:=\left(\begin{array}{ccc}
0 & 0 & 0 \\
0 & 1 & 1 \\
1 & 0 & 2 \\
2 & 2 & 0 \\
3 & 3 & 3 \\
\vdots & \vdots & \vdots \\
h-2 & h-2 & h-2
\end{array}\right)
$$

belongs to $\mathcal{M}\left(\mathbf{k}^{h} \backslash \Gamma_{h}, \mathcal{D}_{\gamma}\right)$ but $\gamma(A)=(0,1, \ldots, h-1) \notin \mathbf{k}^{h} \backslash \Gamma_{h}$, thus $\gamma \notin$ $\operatorname{Pol}\left(\mathbf{k}^{h} \backslash \Gamma_{h}\right)$. We show that $\gamma \in \operatorname{Pol}\left(\zeta_{n}\right)$. Let $B=\left(b_{i j}\right) \in \mathcal{M}\left(\zeta_{n}, \mathcal{D}_{\gamma}\right)$ have $n_{0}$ rows $(0,0,0), n_{1}$ rows $(0,1,1), n_{2}$ rows $(1,0,2), n_{3}$ rows $(2,2,0)$ and $m_{i}$ rows $(i, i, i)$, $i=3, \ldots, h-2$. Consider the case $n_{0}=0$. Observe that then there is a selfmap $\varphi$ of $\mathbf{h}$ such that $\varphi\left(b_{i 1}\right)=c_{i}$ for all $i=1, \ldots, n$. This means that $b_{i 1}=b_{j 1} \Rightarrow c_{i}=c_{j}$ for all $1 \leqslant i<j \leqslant n$. From the definition of $\zeta_{n}$ and $\left(b_{11}, \ldots, b_{n 1}\right) \in \zeta_{n}$, we obtain the required $\gamma(B) \in \zeta_{n}$. The same argument applies if $n_{i}=0$ for some $1 \leqslant i \leqslant 3$ (with the $i$-th column instead of the first one). It remains to consider the case when all $n_{0}, \ldots, n_{3}$ are positive. However, then the first column of $B$ contains $0,1,2$, and so does not belong to $\zeta_{n}$. This concludes the proof of $\gamma \in \zeta_{n}$ and of the proposition.

REMark 17. An infinite independent family of partial clones under $\mathrm{Pol}\left(R_{1}\right)$ and contained in no other maximal partial clone is missing. However, we have a countably infinite chain under this maximal partial clone:

Let $\mathcal{F}$ be the set of all equivalence relations on $2 h:=\{0, \ldots, 2 h-1\}$ with two equivalence classes of even size, and for $n \geqslant 2$, define the $2 n$-ary relation

$$
\tau_{n}:=\bigcup_{e \in \mathcal{F}} \Delta_{e}
$$

Hence $\left(x_{1}, \ldots, x_{2 n}\right) \in \tau_{n}$ if and only if either $x_{1}=\cdots=x_{2 n}$ or there are $a, b \in \mathbf{k}$ such that $\left\{x_{1}, \ldots, x_{2 n}\right\}=\{a, b\}$ and $a$ appears in an even number of times. Clearly $S_{\mathbf{k}} \subseteq \operatorname{Pol}\left(\tau_{n}\right)$ for all $n \geqslant 2$. 
Now as

$$
\tau_{2 n}=\left\{\left(x_{1}, x_{2}, \ldots, x_{2 n-1}, x_{2 n}\right) \mid\left(x_{1}, x_{1}, x_{1}, x_{2}, \ldots, x_{2 n-1}, x_{2 n}\right) \in \tau_{2 n+2}\right\}
$$

we have that

$$
\operatorname{Pol}\left(\tau_{4}\right) \supseteq \operatorname{Pol}\left(\tau_{6}\right) \supseteq \operatorname{Pol}\left(\tau_{8}\right) \ldots
$$

Note that $\tau_{4}=R_{1}$. We show that no equality holds in (1). For $\operatorname{Pol}\left(\tau_{2 n+2}\right) \neq \operatorname{Pol}\left(\tau_{2 n}\right)$, consider the $2 n$-ary partial operation $\psi_{n}$ defined by setting:

$\mathcal{D}_{\psi_{n}}:=\{(0, \ldots, 0),(1, \ldots, 1)\} \cup\{(1,0, \ldots, 0),(0,1, \ldots, 0), \ldots,(0,0, \ldots, 1)\}, \quad$ and $\psi_{n}\left(x_{1}, x_{2}, \ldots, x_{2 n}\right)=0$ if either $\left(x_{1}, x_{2}, \ldots, x_{2 n}\right)$ is a row of the $2 n \times 2 n$ identity matrix or $\left(x_{1}, \ldots, x_{n}\right)=(0,0, \ldots, 0)$ and $\psi_{n}(1,1, \ldots, 1)=1$.

Let $A$ be the $(2 n+2) \times 2 n$ matrix whose first $2 n$ rows form the $2 n \times 2 n$ identity matrix and the last two rows are $(0,0, \ldots, 0)$ and $(1,1, \ldots, 1)$. Now as $A \in \mathcal{M}\left(\tau_{n+1}, \mathcal{D}_{\psi_{n}}\right)$ but $\psi_{n}(A)=(0, \ldots, 0,1) \notin \tau_{n+1}$, we deduce that $\psi_{n} \notin \operatorname{Pol}\left(\tau_{n+1}\right)$. We claim that $\psi_{n} \in \operatorname{Pol}\left(\tau_{n}\right)$. Indeed let $B \in \mathcal{M}\left(\tau_{n}, \mathcal{D}_{\psi_{n}}\right)$ have $m_{1}$ rows $(1,0, \ldots, 0), \ldots, m_{2 n}$ rows $(0, \ldots, 1), t_{1}$ rows $(0, \ldots, 0)$ and $t_{2}$ rows $(1, \ldots, 1)$. We show that $t_{2}$ is even. Suppose to the contrary that $t_{2}$ is odd. Since the columns of $B$ are in $\tau_{n}$, clearly $m_{i}+t_{2}$ is even and therefore $m_{i}$ is odd for all $i=1, \ldots, 2 n$. Moreover $2 n=m_{1}+\cdots+m_{2 n}+t_{1}+t_{2} \geqslant 2 n+t_{1}+1 \geqslant 2 n+1$, a contradiction. Thus $t_{2}$ is even and $\psi_{n}(B)=\left(c_{1}, \ldots, c_{2 n}\right)$ is a zero-one vector with $c_{1}+\cdots+c_{2 n}=t_{2}$, hence $\psi_{n}(B) \in \tau_{n}$.

Next we show that $\mathrm{Pol}\left(\tau_{2 n}\right)$ is contained in no other maximal partial clone on $\mathbf{k}$. Indeed denote by $f$ the partial binary operation with $\mathcal{D}_{f}:=\mathbf{2}^{2}$ and $f(00)=f(1,1):=$ $0, f\left(\begin{array}{ll}0 & 1\end{array}\right)=f(10):=1$ (thus $f$ is the sum $\bmod 2$ with domain $\{0,1\}$ ). We show that $f \in \operatorname{Pol}\left(R_{2}\right)$. Let $A \in \mathcal{M}\left(\tau_{n}, \mathcal{D}_{f}\right)$ have $n_{0}$ rows $(0,0), n_{1}$ rows $(0,1), n_{2}$ rows $(1,0)$ and $n_{3}$ rows $(1,1)$. As the columns of $A$ belong to $\tau_{n}$, we have that $n_{1}+n_{3} \equiv$ $n_{2}+n_{3} \equiv 0(\bmod 2)$, whence $n_{1} \equiv n_{2} \equiv n_{3}(\bmod 2)$. Now $f(A)=\left(c_{1}, \ldots, c_{2 n}\right)$ where $c_{1}+\cdots+c_{2 n}=n_{1}+n_{2} \equiv 2 n_{1} \equiv 0(\bmod 2)$; consequently $f(A) \in \tau_{n}$ However, the matrix

$$
\left(\begin{array}{ll}
1 & 1 \\
1 & 0 \\
0 & 1 \\
0 & 0
\end{array}\right)
$$

can be used to show that $f \notin \operatorname{Pol}\left(R_{2}\right)$. Furthermore, we can use the same partial operations defined in Proposition 16 to show that none of the maximal partial clones $\operatorname{Pol}\left(\Gamma_{k}\right), \operatorname{Pol}\left(\Gamma_{k} \cup \Delta_{k}\right), \operatorname{Pol}\left(\mathbf{k}^{h} \backslash \Delta_{h}\right), 3 \leqslant h \leqslant k, \operatorname{Pol}\left(\Gamma_{4} \cup R_{1}\right)$ contains the partial clone $\operatorname{Pol}\left(\tau_{n}\right)$. 


\section{REFERENCES}

[1] F. Börner, L. Haddad and R. Pöschel, 'Minimal partial clones', Bull. Austral. Math. Soc. 44 (1991), 405-415.

[2] R.V. Freivald, 'Completness criteria for functions of the algebra of logic and many-valued logics', Dokl. Akad. Nauk. SSSR 6 (1966), 1249-1250.

[3] L. Haddad and I.G. Rosenberg, 'Maximal partial clones determined by areflexive relations', Discrete Appl. Math. 24 (1989), 133-143.

[4] L. Haddad and I.G. Rosenberg, 'Completness theory for finite partial algebras', Algebra Universalis 29 (1992), 378-401.

[5] L. Haddad and I.G. Rosenberg, 'Finite clones containing all permutations', Canad. J. Math. 46 (1994), 951-970.

[6] A.I. Mal'tsev, 'Iterative algebras and Post's varieties', (in Russian), Algebra i Logika 5 (1966), 5-24, English translation: The metamathematics of algebraic systems, Collected papers 1936-67, Stud. Logic. Found. Math. 66, (North-Holland, 1971).

[7] E. Post, The two-valued iterative systems of mathematical logic, Ann. of Math. Studies 5 (Princeton University Press, Princeton, 1941).

[8] B.A. Romov, 'The algebras of partial functions and their invariants', (in Russian), Kibernetika 2 (1981), 1-11; English translation: Cybernetics 17 (1981), 157-167.

[9] B.A. Romov, 'Maximal subalgebras of algebras of partial multivalued logic functions', (in Russian), Kibernatika 1 (1980), 31-40; English translation, Cybernetics 16 (1980) 28-36.

Département de Mathématiques et Informatique Collège Militaire Royal du Canada

Kingston, Ontario, K7L 5LO

Canada
Département de Mathématiques et Statistique

Université de Montréal

C.P. 6128 Succ Centreville

Montréal, Qué, H3C 3J7

Canada 